\title{
Macular hole surgery with and without indocyanine green assistance
}

\begin{abstract}
Purpose To compare the results of macular hole surgery with the use of indocyanine green (ICG) to assist internal limiting membrane (ILM) peeling and macular hole surgery without indocyanine green use.
\end{abstract}

Methods A retrospective, comparative, consecutive study of 68 patients with macular holes. In total, 34 patients underwent vitrectomy and ILM peeling, and 34 patients underwent vitrectomy and ILM peeling with the assistance of indocyanine green. The main outcome measures were postoperative visual acuity and macular hole status.

Results Indocyanine green increased the ability to visualise and peel the ILM. The average preoperative visual acuity in the group where ICG was used was $6 / 36$ and the group where ICG was not used was $6 / 60$. The average follow-up was 25 weeks for the ICG group and 53 weeks for the group with no ICG. Both groups were also compared at 25 weeks follow-up. Hole closure rate for the group with ICG was $97 \%$ compared to $91 \%$ without ICG. The mean postoperative visual acuity was 6/24 for the group with ICG and 6/12 for the group without ICG, a difference of two lines on the Snellen chart when compared with the preoperative acuity ( $P$-value 0.299 , Student's $t$-test). Both groups had a mean improvement of Snellen acuity of two lines (ICG group: $P$-value $\mathbf{0 . 0 0 0 2}$, Student's $\boldsymbol{t}$-test; no ICG group: $P$-value $\mathbf{0 . 0 0 0 0 4}$, Student's $t$-test). In all, $83 \%$ of patients in the ICG group maintained or improved their visual acuity compared to $91 \%$ in the group without ICG. Discussion There is no doubt that indocyanine green stains and assists in visualisation and therefore increases the ease of peeling the ILM in macular hole surgery. Initially, there was concern regarding a poorer outcome for patients with the use of ICG, which has also been previously discussed in
K Slaughter and IL Lee

the literature. When the two groups were compared at a similar follow-up time of approximately 25 weeks, it was shown that there was no statistically significant difference between the outcomes in the two groups. This study had an improved hole closure rate for the group where ICG was used, although it was not statistically significant. Eye (2004) 18, 376-378. doi:10.1038/ sj.eye. 6700666

Keywords: indocyanine; green; macular hole; internal limiting membrane

\section{Introduction}

Prior to 1990, there was no treatment for macular holes. The surgical treatment for macular holes was first reported by Kelly and Wendel $^{1}$ in 1991. Many investigators subsequently reported the use of vitrectomy with adjuvent therapies including transforming growth factor, concentrated platelets, and autologous serum. ${ }^{2-3}$ Vitrectomy and internal limiting membrane (ILM) peeling were first described in $1998 .{ }^{4}$ Indocyanine green (ICG) was reported in 2000 to stain ILM in cadavers. ${ }^{5}$

Kadonosono et $a l^{6}$ reported on 13 patients who received ICG to assist peeling of the ILM. They felt that ICG allowed safer and easier removal of the ILM. Further groups have shown that ICG stains and increases the ease of peeling the ILM. ${ }^{7-9}$ To date, there are no large studies using ICG and no large studies comparing the results of the use of ICG to no ICG. Recent literature has expressed concern regarding the outcome in patients where ICG has been used. ${ }^{4,10}$ Gandofer et al ${ }^{10}$ studied the histology of the ILM after removal with the assistance of ICG, and felt that ICG may alter the structure of the retina. Sippy et $a l^{4}$ did a laboratory experiment on cultured RPE cells and demonstrated that ICG resulted in decreased mitochondrial enzyme activity of the retina but did not alter the structure. 
The aim of this study was to compare the outcome results of patients where ICG had been used to assist peeling the ILM in macular hole surgery to patients where ICG had not been used.

\section{Methods}

The records of 68 patients who had undergone macular hole surgery by a single surgeon were reviewed. The first 34 had undergone macular hole surgery with ILM peeling aided by ICG and the other 34 without the aid of ICG. ICG has recently been reported to enhance the ease of peeling the ILM in macular hole surgery. Encouraged by these reports, the author of this series changed his technique to utilise ICG. There were no exclusions. In this paper, these two groups will be referred to as the no ICG group and the ICG group, respectively.

\section{Surgical technique}

All patients underwent macular hole repair with peeling of the ILM. The procedure included a three port pars plana vitrectomy. The central vitreous was removed initially, followed by peeling of the posterior hyaloid face from the optic disc and posterior retina. Where ICG was used, it was injected in solution over the macular region. The infusion was temporarily stopped for $30 \mathrm{~s}$ and when it was restarted the excess ICG was removed with a flute needle. A bent sclerotomy blade was used to incise the ILM, an edge was lifted, and then the ILM was peeled with intraocular forceps in a circumferential manner around the macular hole. A fluid air exchange was then performed aspirating over the optic disc but not over the macular hole. An air gas exchange was performed using perfluoropropane $\left(\mathrm{C}_{3} \mathrm{~F}_{8}\right)$ 10-12\%.

The sclerotomies were closed, and subconjunctival dexamethasone and gentamicin were given with minims atropine. The patients commenced a face down position on return to the recovery room and stayed positioned face down for 2 weeks following surgery (at least $45 \mathrm{~min}$ every hour).

The patients' visual acuity and macular hole status were recorded at each postoperative visit. Snellen visual acuity was converted to a logmar scale for comparison.

\section{Statistical analysis}

Student's two-tailed unpaired $t$-tests were used to test for significance between the two groups. A $P$-value of less than 0.05 was considered significant.

\section{Results}

The average age for the ICG group ranged from 53 to 85 years of age with an average of 67.5 years. The average age for the no ICG group was 66.2 years with a range from 16 to 80 years. All macular holes in both groups ranged from stage 2 to 3 and were operated on within 1 year of presentation. All the no ICG patients underwent a vitrectomy with peel of ILM, with one patient undergoing a combined vitrectomy, peel, and cataract extraction with IOL. In the ICG group, six cases underwent the combined procedure of vitrectomy, peeling of ILM, and phacoemulsification of cataract with a posterior chamber intraocular lens.

In the no ICG group, 20 patients had macular holes involving the left eye and 14 had macular holes involving the right eye. Nine were male and 25 were female. In total, 30 were phakic preoperatively and at final followup 16 were phakic. The total follow-up was 53.5 weeks. The ICG group had 15 patients with left eye macula holes and 19 patients with right eye macula holes. In all, 10 were male and 24 female. In total, 26 were phakic preoperatively and 16 were phakic at follow-up. Therefore, both groups had 16 patients who were phakic at final follow-up. All holes were idiopathic apart from one in a 16-year-old boy, which occurred following trauma. The total follow-up was 25.4 weeks. Both groups were also compared at a follow-up of approximately 25 weeks.

Student's unpaired two-tailed $t$-tests revealed no significant difference between the ICG and no ICG group at follow-up of 25 weeks ( $P=0.299)$, or total follow-up. There was a statistically significant difference for the groups when patients were compared preoperatively to postoperatively $(P=0.0004$ for no ICG, $P=0.0002$ for ICG group).

The visual acuity preoperatively in the ICG group had an average of $6 / 36$ or -0.78 logmar, the no ICG group had an average of $6 / 60$ or -1 logmar. The average visual acuity postoperatively in the ICG group was 6/24 (-0.6 logmar) and in the no ICG it was 6/12 $(-0.3)$ at final follow-up (Figure 1). When the two groups were compared at 25 weeks, the average visual acuity was $6 / 24$ (-0.6 logmar) for both groups (Figure 2). The hole

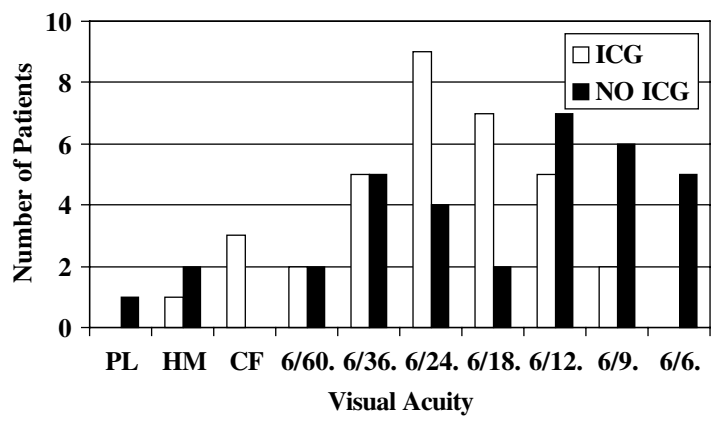

Figure 1 Comparing visual acuity postoperatively at final follow-up: ICG $=25$ weeks and $\mathrm{nO}$ ICG $=53$ weeks. 


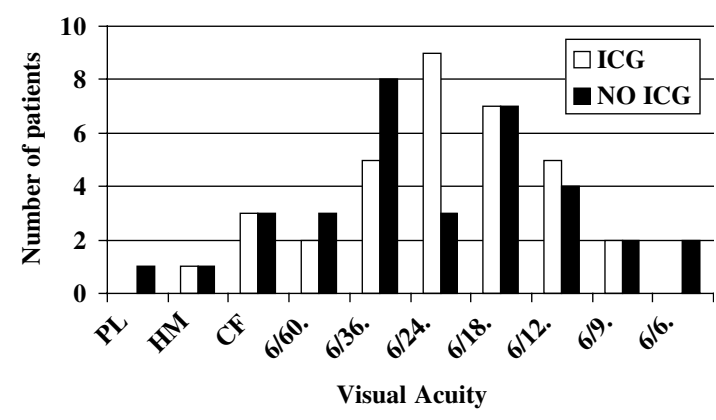

Figure 2 Comparing visual acuity for ICG group and no ICG group at 25 weeks follow-up.

closure rate was $97 \%$ in the ICG group and $91 \%$ in the no ICG group at primary operation. Using the $\chi^{2}$ test, the difference was not significant, although maybe with larger patient numbers significance may be shown. In the ICG group, one patient whose macular hole did not close underwent a repeat vitrectomy and peel with ICG, which successfully closed the macula hole. Therefore, at second operation the ICG group had a 100\% hole closure rate. At reoperation of this case, ICG was used again and a few fibres stained; these were peeled and considered to be possible ILM remnants or fine epiretinal membrane fibres. The no ICG group had three of 34 patients who after the first operation had an open hole. The first developed endophthalmitis, the second had a retinal detachment, and the third underwent a second peel without ICG, which was successful. Therefore, there was a $94 \%$ hole closure rate at reoperation for the no ICG group. No postoperative retinal pigment epithelial changes were noted in the patients where ICG was used.

\section{Discussion}

Concerns have been raised in recent literature of the safety of ICG in ILM peeling for macular hole surgery.,10 Initially, in this study, it was felt that the group of patients where ICG had been used were not achieving the same visual acuity as the patients where ICG had not been used. We were initially comparing the no ICG group at follow-up of 53 weeks to the ICG group with a follow-up of 25 weeks. There was a two-line Snellen difference in visual acuity between the two groups. When we compared the data at a similar follow-up time of 25 weeks, it was found that the visual acuity was approximately the same for the two groups (average $=$ 6/24) (Figure 2). Therefore, as the group where ICG was not used continued to improve their visual acuity to 53 weeks, we hypothesise that the group receiving ICG will continue to improve with further follow-up. This needs to be evaluated with further follow-up.
This data shows similar outcomes for patients where ICG has been used when compared to patients where ICG has not been used. We also achieved an improved hole closure rate when ICG was used, although not statistically significant. The increased hole closure rate in patients where ICG has been used could be explained by the increased ease of visualising the ILM and therefore a more complete removal of the ILM, compared with almost blind peeling of the ILM. These results decrease concerns raised in the recent literature, ${ }^{4,10}$ that ICG assisted peeling of the ILM results in a decreased outcome when compared to cases where ICG had not been used. ICG definitely assists in visualisation of the ILM and enhances the ease of the operation as found in previous studies. ${ }^{11}$ An incidental finding was that peeling of the ILM whether with ICG or without had a statistically significant improvement in final visual acuity for patients.

\section{References}

1 Kelly NE, Wendel RT. Vitreous surgery for idiopathic macula holes: results of a pilot study. Arch Ophthalmol 1991; 109: 654-659.

2 Glaser BM, Michels RG, Kupperman BD, Sjaarda RN, Pena RA. Transforming growth factor B2 for the treatment of full thickness macular holes: a prospective randomized study. Ophthalmology 1992; 99: 1162-1172.

3 Liggett PE, Skolik DSA, Horio B, Saito Y, Alfaro V et al. Human autologous serum for the treatment of full thickness macular holes: a preliminary study. Ophthalmology 1995; 102: 1071-1076.

4 Sippy BD et al. Indocyanine green effect on cultured human retinal pigment epithelial cells - implication for macular hole surgery. Am J Opthalmol 2001; 132(3): 433-435.

5 Burk SE, De Mata AP, Synder ME, Rosa RH, Foster RE. Indocyanine green assisted peeling of the retinal internal limiting membrane. Ophthalmology 2000; 107: 2010-2013.

6 Kadononsono K, Itoh N, Uchio E, Nakamura S, Ohno S. Staining of internal limiting membrane in macular hole surgery. Arch Ophthalmol 2000; 118: 1116-1118.

7 Gandorfer A, Messmer EW, Ulbig MW, Kampik A. Indocyanine green selectively stains the internal limiting membrane. Am J Opthalmol 2001; 131: 387-388.

8 Kusaka S, Hayashi N, Ohji M, Hayashi A, Kamei M, Tano Y. Indocyanine green facilitates removal of epiretinal and internal limiting membranes in myopic eyes with retinal detachment. Am J Opthalmol 2001; 131: 388-390.

9 Kwok AH, Li WWY, Pang CP, Lai TYY, Yam GHF et al. Indocyanine green staining and removal of internal limiting membrane in macular hole surgery: histology and outcome. Am J Opthalmol 2001; 132: 178-183.

10 Gandorfer A, Haritoglou C, Gass CA, Ulbig MW, Kampik A. Indocyanine green-assisted peeling of the internal limiting membrane may cause retinal damage. $A m$ J Opthalmol 2001; 132(3): 421-433.

11 Da Mata AP, Burk SE, Riemann CD, Rosa RH, Snyder ME et al. Indocyanine green-assisted peeling of the retinal internal limiting membrane during vitrectomy surgery for macular hole repair. Ophthalmology 2001; 108: 1187-1192. 